\title{
Prunus hybrids rootstocks for flat peach
}

Pilar Legua ${ }^{1 *}$, Jorge Pinochet ${ }^{2}$, María Ángeles Moreno ${ }^{3}$, Juan José Martínez ${ }^{1}$, Francisca Hernández ${ }^{1}$

'Universidad Miguel Hernández - Depto. de Producción Vegetal y Microbiología, Ctra Beniel km, 3.2 - 03312 Orihuela, Alicante - Spain.

${ }^{2}$ Agromillora lberia S.L., C/El Rebato s/n - 08739 -

Subirats, Barcelona - Spain.

${ }^{3}$ CSIC/Estación Experimental de Aula Dei - Depto. de

Pomología, Apdo. 13034 - 50080 - Zaragoza - Spain.

*Corresponding author <p.legua@umh.es>

Edited by: Luís Reynaldo Ferracciú Alleoni

Received October 21, 2010

Accepted June 01, 2011
ABSTRACT: Peach (Prunus persica L.) is the most important stone fruit tree grown in Spain and is the second most important fruit crop in Europe. The influence of eight Prunus rootstocks (GF-677, Krymsk $^{\oplus}$ 86, PADAC 97-36, PADAC 99-05, PADAC 9912-03, PADAC 0024-01, PAC 0021-01 and PAC 0022-01) on vigor, yield and fruit quality traits of 'UFO 3' flat peach cultivar was studied. The highest trunk cross sectional area was exhibited by GF- 677 and the lowest by PADAC 99-05, while intermediate values were found on the other rootstocks. The highest yield efficiency was found on PADAC 99-05, PAC 0021-01, PAC 0022-01 and PADAC 0024-01 and the lowest was shown on $\mathrm{Krymsk}^{\circledR} 86$. The fruit quality parameters measured were color, fruit and stone weights, equatorial diameter, pulp thickness, pulp yield, firmness, $\mathrm{pH}$, soluble solids content and titratable acidity. 'UFO 3' grafted on GF-677 resulted in the largest fruit weight, while the smallest was on PADAC 99-05. Fruits of 'UFO 3' showed a tendency to have higher firmness, higher red colored skin and RI when grafted on PADAC 99-05.

Keywords: SSC, fruit weight, rootstock, acidity, yield

\section{Introduction}

Peach (Prunus persica L.) is the second most important fruit crop in Europe after apple and it is the most important stone fruit tree grown in Spain. In 2009, the Spanish production was $1,225,700$ tons from a surface area of 72,000 ha $(\mathrm{FAO}, 2011)$. Spain is also the third world producer, after China and Italy, and the second in the European Union. Murcia, registering a production of 218,240 tons in 2008 (MARMM, 2009), is the third most important producing region in Spain. This area has a Mediterranean temperate climate, with mild-cold winters and very hot and dry summers.

Traditionally, production and marketing of flat peaches in Spain was limited both in quantity and quality. The main drawbacks of traditional cultivars concern their lack of appealing color, low consistency of the fruits and excessive cracking of the pistil. Thus, the product does not meet market quality standards. Also, marketing is affected by a lack of varieties to properly supply yearround demands. In the last decade, new flat peach cultivars, bearing more attractively colored fruit with good consistency, were introduced into Spain, which has led to an increase in production and marketing. The newly introduced flat peach cultivars came mainly from France (A.S.F.) and Italy (I.S.F. Roma) countries that have not yet experienced the production increase that has occurred in Spain.

Calcareous soil conditions are among the most limiting abiotic stresses in this area. Consequently, the most commonly used rootstocks are those derived from peach-almond ( $P$. persica $\times P$. amygdalus $)$ and peach $\times P$. davidiana hybrids. New rootstocks recently released or under selection allow peach cultivation in different soils and climatic conditions, and provide tolerance and/or resistance to trees against pests and diseases (Cinelli and Loreti, 2004; Dichio et al., 2004). Although commercial peach cultivars are usually grafted on different rootstocks and information is available on its influence on vigor, graft-compatibility and yield characteristics (Bielicki et al., 2004; Giorgi et al., 2005; Hudina et al., 2006; Iglesias et al., 2004; Zarrouk et al., 2005; Zarrouk et al., 2006) very few studies refer to the influence of the rootstock over fruit quality parameters in the grafted scion (Albás et al., 2004; Caruso et al., 1996; Remorini et al., 2008; Orazem et al., 2011) and none are available for flat peaches. Therefore, the information provided in this research is regarded of high interest for the flat peach producers, cooperatives as well as commercial channels.

Nowadays breeders are not only interested in productivity, but also in better fruit quality (Byrne, 2002; Cevallos-Casals et al., 2006) and specially in the case of flat peaches, where income is strongly associated with high content of soluble sugars and fruit size. The influence of rootstock on the fruit quality has been reported by Bielicki et al. (2000, 2004); Caruso et al. (1996), Caruso et al. (1997), Chun and Fallahi (2001) and Cantín et al. (2009). The three most important components in the organoleptic quality of fruit are aroma, sugar content and acidity, which are related to many chemical and physical properties of fruits (Crisosto et al., 2003), and these properties are highly influenced by rootstocks. The aim of this work is to analyse the influence of eight different Prunus rootstocks on several agronomical and fruit quality parameters of the flat peach cultivar 'UFO 3'.

\section{Materials and Methods}

Eight Prunus rootstocks were compared in one trial during a three year period (2006-2008). They were established in the field in the winter of 2003-2004, and grafted in situ with the flat peach 'UFO 3' [Prunus persica (L.) Batsch var. platycarpa] during the summer of 2004. This cultivar was obtained in Rome, Italy. It has a flat shape, sub-acid taste, white flesh and early maturation (Nicotra and Conte, 2003). The choice of this cultivar 
was due to the high interest in this type of material in south-eastern Spain, and because of its early maturation and good fruit quality.

Most of the rootstocks under evaluation are experimental interspecific hybrids (PADAC and PAC series), tolerant to iron-chlorosis and resistant to nematodes (Table 1). None of the studied rootstocks emitted suckers. The peach-almond hybrid GF-677 was used as the reference rootstock because it is the most commonly widespread rootstock for peach in the Mediterranean area.

The trial was carried out at an experimental orchard located near Cieza, Murcia, Spain $\left(38^{\circ} 17^{\prime} \mathrm{N} ; 1^{\circ} 27^{\prime}\right.$ W). Soil conditions were poor and stony in the first layer $(0-10 \mathrm{~cm})$ and clayish below this level (Calcaric Regosols). Soil was very calcareous, with $15 \%$ content of active lime and $\mathrm{pH}=8.5$. Trial was drip irrigated. Trees were trained to the central leader system and planted at a spacing of $4 \mathrm{~m} \times 2.5 \mathrm{~m}$. Standard cultural practices (pruning, thinning, fertilization and treatments) were performed. The experiment was established in a randomized block design with five single-tree replications for each scionstock combination. Guard rows were used to minimize edge effects. Vegetative traits were evaluated over three consecutive years (2006-2008) and fruit quality over two years (2007-2008).

The following plant parameters were measured and/or calculated: trunk circumference at $10 \mathrm{~cm}$ above the graft union, the total yield and yield efficiency. The trunk circumferences were converted into trunk crosssectional areas (TCSA). Cumulative yield per tree and yield efficiency of each scion-stock combination were computed from the harvest data. The plant yield efficiency was expressed as the ratio of total cumulative yield in kg per final TCSA.

Fruits were sampled for analyses when the first commercial harvest took place in two consecutive years. Harvest criteria were based mainly on firmness, color development and solid soluble contents for each rootstock combination. Harvesting date ranged from $2^{\text {th }}$ to $6^{\text {th }}$ May, depending on the year. Fruit from each variety/ rootstock combination was randomly harvested from five trees and 20 representative fruits reaching maturity were processed for all the analyses. Peaches were harvested from parts of the trees to avoid fruit position effect (Taylor et al., 1993).
Fruit weights were determined with a 0.01 g-accuracy balance. Equatorial diameter and pulp thickness were measured with a $0.01 \mathrm{~mm}$-accuracy electronic digital slide gauge. Two readings per fruit were made according to UPOV (1995) protocol for peaches. Pulp yield percentage was also calculated as [/fruit weight-stone weight)/fruit weight] $\times 100$. Fruit firmness was measured on opposite sides of the equator of each fruit with a Bertuzzi penetrometer fitted with an 8-mm diameter probe (model FT-327, Facchini, Alfonsine, Italy). Two readings were averaged for each fruit. Color determinations were made for fruit skin on four opposite faces in the equatorial zone. Values of the CIELAB $L^{*}$ (brightness or lightness; $0=$ black, $100=$ white $), a^{*}\left(-a^{*}=\right.$ greenness, $+a^{*}=$ redness $)$ and $b^{*}\left(-b^{*}=\right.$ blueness, $+b^{*}=$ yellowness) color variables were measured using a chromatometer.

The chemical analyses were determined using two juice samples (ten peaches each) for each scion/stock combination. The juice was extracted by macerating the fruit pulp in a commercial blender and filtered through fine opening cheesecloth. The $\mathrm{pH}$ was measured with a pH-meter micropH 2001 (Crison). The soluble solids content (SSC) of the juice was measured with a temperature compensated refractometer (model N-1, Atago Co., Tokyo, Japan); and data were given as ${ }^{\circ} \mathrm{Brix}\left( \pm 0.2^{\circ} \mathrm{Brix}\right.$ at $20^{\circ} \mathrm{C}$ ). The titratable acidity (TA) was determined by titration with $0.1 \mathrm{~mol} \mathrm{~L}^{-1} \mathrm{NaOH}$ to $\mathrm{pH} 8.1$. Data were given as $\mathrm{g} \mathrm{L}^{-1}$ malic acid, since this is the dominant organic acid in peach. The ripening index (RI) was calculated as the ratio of SSC/TA.

Statistical analyses were performed using SPSS Software, v.16.0 for Windows. A basic descriptive statistical analysis was followed by an analysis on variance test for mean comparisons. The method used to discriminate among the means (Multiple Range Test) was the Fisher's Least Significant Difference (LSD) procedure $(p<0.05)$. Correlation analyses between traits to reveal possible relationships were also carried out over the three years.

\section{Results and Discussion}

Tree size, as assessed by TCSA, was affected by rootstock. The greatest TCSA was exhibited with GF-677 and the lowest with PADAC 99-05, while intermediate

Table 1 - Rootstocks used in the present study.

\begin{tabular}{|c|c|c|}
\hline Rootstock & Species & Origin \\
\hline GF-677 & Prunus persica $\times$ Prunus amygdalus & INRA $^{* * *}$, Bordeaux, France \\
\hline Krymsk $^{\circledast} 86$ & Prunus cerasifera $\times$ P. persica & Krymsk Breeding Station, Krasnodar, Russian Federation \\
\hline PADAC 97-36 & Prunus cerasifera $\times(P$. persica $\times P$. dulcis $)$ & Co-obtention between $\mathrm{Al}^{*}$ and $\mathrm{CSIC}{ }^{* *}$, Spain \\
\hline PADAC 99-05 & Prunus cerasifera $\times(P$. persica $\times$ P. dulcis $)$ & Co-obtention between $\mathrm{Al}^{*}$ and $\mathrm{CSIC}^{* *}$, Spain \\
\hline PADAC 9912-03 & Prunus amygdalus $\times$ P. persica & Co-obtention between $\mathrm{Al}^{*}$ and $\mathrm{CSIC}^{* *}$, Spain \\
\hline PADAC 0024-01 & P. persica $\times(P$. dulcis $\times$ P. persica $)$ & Co-obtention between $\mathrm{Al}^{*}$ and $\mathrm{CSIC}^{* *}$, Spain \\
\hline PAC 0021-01 & (P. dulcis $\times$ P. persica $) \times P$. persica & Agromillora Iberia, Spain \\
\hline PAC 0022-01 & $(P$. dulcis $\times$ P. persica $) \times P$. salicina & Agromillora Iberia, Spain \\
\hline
\end{tabular}


values were found on the other rootstocks (Table 2). The increasing rate of TCSA was highly affected by rootstocks, being higher for GF-677 (65 $\mathrm{cm}^{2}$ per year) and lower for PADAC 99-05 (35 $\mathrm{cm}^{2}$ per year). This last rootstock exhibited an increased capacity to control tree size, a trait that would seem ideal for high density orchards allowing an important reduction in labor costs.

In the first bearing years (2006-2007), yields were very low, and there were no rootstock differences (data not shown). However, differences among rootstocks became evident in the $5^{\text {th }}$ year after grafting (Table 2). Thus, trees grafted on GF-677, PADAC 0024-01, PAC 0021-01 and PAC 0022-01 had the highest yields, while Krymsk $^{\circledR} 86$ induced the lowest yield. The rootstock can influence tree size and production in the grafted cultivars. In the present study GF-677 had a high productive potential, similar to "Suncrest" (a common yellow-pulp peach) grafted onto GF-677 (Giorgi et al., 2005).

By year fifth after grafting, the cumulative yield was greater on GF-677 (Table 2). In contrast, Krymsk ${ }^{\circledR}$ 86 induced the lowest cumulative yield. The greatest yield efficiency was found on PADAC 99-05, PAC 002101, PAC 0022-01 and PADAC 0024-01 and the lowest was shown on 'Krymsk ${ }^{\circledR} 86$ ', although they did not dif-

Table 2 - Effect of rootstock on TCSA (trunk cross-sectional area), yield, cumulative yield and yield efficiency of the flat peach 'UFO $3^{\prime}$ in the $5^{\text {th }}(2008)$ year after grafting.

\begin{tabular}{|c|c|c|c|c|}
\hline Rootstock & TCSA & Yield 2008 & $\begin{array}{c}\text { Cumulative yield } \\
2006-2008\end{array}$ & $\begin{array}{c}\text { Yield } \\
\text { efficiency }\end{array}$ \\
\hline & $\mathrm{cm}^{2}$ & \multicolumn{2}{|c|}{ - kg per tree -} & $\mathrm{kg} \mathrm{cm}^{-2}$ \\
\hline GF-677 & $124.57 \mathrm{c}$ & $18.57 \mathrm{~d}$ & $57.03 \mathrm{e}$ & $0.17 a b$ \\
\hline Krymsk $^{\circledast} 86$ & $68.56 \mathrm{~b}$ & $6.57 \mathrm{a}$ & & $0.13 a$ \\
\hline PADAC 97-36 & 6 & & & $0.20 a b$ \\
\hline PADAC & a & 18 bc & & $0.33 b$ \\
\hline PADAC 9912-03 & & & & $0.22 a b$ \\
\hline PADAC 0024-01 & & $16.23 \mathrm{~cd}$ & & $0.29 \mathrm{~b}$ \\
\hline PAC 0021-01 & $73.04 b$ & $17.06 \mathrm{~cd}$ & $51.13 \mathrm{de}$ & $0.32 \mathrm{~b}$ \\
\hline PAC 0022-01 & $77.46 \mathrm{~b}$ & $15.23 \mathrm{bcd}$ & $45.70 \mathrm{~cd}$ & $0.29 \mathrm{~b}$ \\
\hline
\end{tabular}

For each character, means followed by the same letter in each column are not different (LSD test, $p<0.05$ ). fer from GF-677, PADAC 97-36 and PADAC 9912-03. The poor performance of 'Krymsk ${ }^{\circledR} 86$ ' was probably due to its bad adaptation to the growing conditions that prevail in Murcia. This peach-plum hybrid is from Russia originally, where chill requirements are much higher than those found in a warm Mediterranean environment where this study was conducted. In spite of the tendency of GF-677 to show higher yields, its higher vigor could limit its yield efficiency.

Fruit weight and size were affected by rootstock (Table 3). The highest fruit weight was induced by GF677 , the most invigorating rootstock, while PADAC 9905 (lowest vigor) induced the lowest fruit weight in the two years of study. Our results for GF-677 fruit weights are discrepant with those obtained by Ozarem et al. (2011). Growing conditions and cultural practices could account for these differences. Regarding the fruit size, the largest equatorial diameter was also found onto GF677. In contrast, the smallest equatorial diameter was recorded on PADAC 99-05, although this did not differ from Krymsk ${ }^{\circledR} 86$ in the two years of study. The results obtained for GF-677 in this study are superior to those obtained by Giorgi et al. (2005). Mean fruit weight and caliber of 'UFO 3' were superior to those reported by Nicotra et al. (2001) for all tested rootstocks with the exception of Krymsk ${ }^{\circledR} 86$ and PADAC 99-05. The tendency of PADAC 99-05 to produce lower fruit weights seems to be associated by its capacity to control vigor, suggesting that fruit size is also affected. Another explanation could be due to sink competition among fruits by the assimilate supply. Thus, fruit thinning should probably be stronger on this rootstock.

'UFO 3 ' had the lowest pulp thickness when grafted on PADAC 99-05, Krymsk ${ }^{\circledR}$ 86, PAC 0021-01 and PAC 0022-01, whereas GF-677, PADAC 97-36, PADAC 9912-03 and PADAC 0024-01 had the highest mean values for pulp thickness and smaller size seeds (data not shown), thus presenting the highest pulp yields (Table 3). The best pulp yield was found on GF-677 but it did not differ from PADAC 97-36, PADAC 9912-03 and PADAC 0024-01, while the lowest pulp yield was recorded

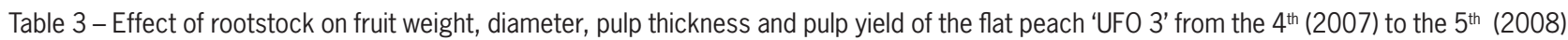
year after grafting.

\begin{tabular}{|c|c|c|c|c|c|c|c|c|c|c|c|c|}
\hline \multirow{2}{*}{ Rootstock } & \multicolumn{3}{|c|}{ Fruit weight } & \multicolumn{3}{|c|}{ Equatorial diameter } & \multicolumn{3}{|c|}{ Pulp thickness } & \multicolumn{3}{|c|}{ Pulp yield } \\
\hline & 2007 & 2008 & Average & 2007 & 2008 & Average & 2007 & 2008 & Average & 2007 & 2008 & Average \\
\hline & \multicolumn{3}{|c|}{$-\mathrm{g} \longrightarrow$} & \multicolumn{6}{|c|}{$\longrightarrow \mathrm{mm} \longrightarrow$} & \multicolumn{3}{|c|}{ $\%$} \\
\hline GF-677 & $86.82 \mathrm{e}$ & $112.70 \mathrm{~d}$ & 99.76 e & $61.40 \mathrm{~d}$ & $68.78 d$ & $65.09 \mathrm{~d}$ & $21.75 d$ & $23.21 a b$ & $22.48 \mathrm{c}$ & $96.43 a b c$ & 95.14 e & $95.42 \mathrm{~d}$ \\
\hline Krymsk $^{\circledR} 86$ & $71.10 \mathrm{ab}$ & 97.33 bc & $84.21 b$ & $57.93 \mathrm{ab}$ & $64.23 \mathrm{ab}$ & $61.08 \mathrm{ab}$ & $19.04 a b$ & $22.05 \mathrm{a}$ & $20.54 \mathrm{a}$ & $95.93 \mathrm{a}$ & 94.28 bc & $94.63 \mathrm{~b}$ \\
\hline PADAC 97-36 & $80.34 \mathrm{~cd}$ & 93.06 b & & $59.58 \mathrm{bc}$ & $65.74 b c$ & $62.66 \mathrm{c}$ & $20.57 c$ & 24.08 b & $22.32 \mathrm{bc}$ & 96.0 & $94.91 \mathrm{de}$ & $95.16 \mathrm{~cd}$ \\
\hline PADAC 99-05 & $66.50 \mathrm{a}$ & 86.03 a & $76.26 \mathrm{a}$ & $56.28 \mathrm{a}$ & $63.01 \mathrm{a}$ & $59.65 \mathrm{a}$ & $17.99 \mathrm{a}$ & $22.85 \mathrm{ab}$ & $20.42 \mathrm{a}$ & $96.09 a b$ & 93.58 a & $94.12 \mathrm{a}$ \\
\hline PADAC 9912-03 & $77.63 c$ & $91.64 \mathrm{ab}$ & $84.63 b c$ & $60.62 \mathrm{~cd}$ & $66.19 c$ & $63.40 \mathrm{c}$ & $20.19 b c$ & $24.20 b$ & $22.19 b c$ & $96.69 \mathrm{c}$ & $94.74 \mathrm{de}$ & $95.16 \mathrm{~cd}$ \\
\hline PADAC 0024-01 & $85.38 \mathrm{de}$ & $103.29 \mathrm{c}$ & $94.34 \mathrm{de}$ & $61.36 \mathrm{~d}$ & $65.75 b c$ & $63.56 c$ & $20.64 \mathrm{~cd}$ & $24.19 b$ & $22.41 \mathrm{c}$ & 96.64 bc & $94.56 \mathrm{~cd}$ & $95.01 \mathrm{bcd}$ \\
\hline PAC 0021-01 & $78.01 \mathrm{c}$ & $102.00 \mathrm{c}$ & $90.00 \mathrm{~cd}$ & 59.31 bc & 65.16 bc & $62.23 b c$ & $19.50 \mathrm{bc}$ & 23.01ab & $21.25 a b$ & $96.70 \mathrm{c}$ & 94.26 bc & 94.79 bc \\
\hline PAC 0022-01 & $74.55 \mathrm{bc}$ & $100.28 \mathrm{c}$ & 87.42 bc & 58.76 b & $66.38 c$ & $62.57 b c$ & $19.11 \mathrm{ab}$ & $23.85 b$ & $21.48 \mathrm{abc}$ & $96.77 \mathrm{c}$ & $94.01 \mathrm{ab}$ & $94.60 a b$ \\
\hline
\end{tabular}

For each character, means followed by the same letter in each column are not different (LSD test, $p<0.05$ ). 
on PADAC 99-05. This is probably due to their low fruit weight and high stone weight.

Fruit firmness of 'UFO 3' was affected by the rootstock (Table 4). The highest firmness was found on the less vigorous rootstock PADAC 99-05, although it did not differ from GF-677, PADAC 9912-03 and PADAC 0024-01. These results would agree with those obtained by Tsipouridis and Thomidis (2005), who reported the tendency of GF-677 to induce the highest fruit firmness when compared with other peach rootstocks. This implies a better resistance of fruit to post-harvest damage. The lowest value was recorded on PAC 0021-01, although the differences were not significant when compared with PAC 0022-01. The rest of rootstocks induced intermediate firmness values. Several dwarfing rootstocks induced higher firmness in sweet cherry cultivars (Gonçalves et al., 2006), as it was found in this work with the least invigorating rootstock PADAC 99-05.

Fruit color is an important factor for marketability and consumer acceptance and can also be indicative of the ripening index. Fruit color intensity of peaches is directly associated with appearance and affects consumer acceptance and sales, and this is why new cultivars released over the last few decades initiate red coloration of the fruit skin at an early stage and develop and intense red color (Iglesias and Echeverría,
2009). The color of 'UFO 3 ' fruit was affected by the rootstock (Table 4). The brightest colored skin (high L* value) was found in fruits onto PAC 0021-01 without being different from GF-677, whereas the darkest colored skin was found on PADAC 99-05, PADAC 9912-03, Krymsk $^{\circledR}$ 86, PADAC 97-36, PAC 0022-01 and PADAC 0024-01.

Fruit onto PADAC 99-05 and PADAC 9912-03 had the highest red colored skin (high $\mathrm{a}^{*}$ values). 'UFO 3' grafted on PAC 0021-01 had the most yellow colored skin (high $b^{*}$ and low $a^{*}$ values). The excess of foliage on trees grafted onto PAC 0021-01 seems to have limited sun exposure to maturing fruits resulting in a higher percentage of yellow colored fruits. No differences were found among rootstocks for $\mathrm{a}^{*} / \mathrm{b}$ * ratio (Table 4) with the exception of PAC 0021-01 which showed a lower value (more yellow colored fruits).

Trees grafted on PADAC 99-05 and PADAC 991203 would have redder peaches than fruits from the other rootstocks. Taking into account the color parameters of $a^{*} / b^{*}$ and higher $a^{*}$ values, more attractive fruits would be found on PADAC 99-05 and PADAC 9912-03.

Fruits of 'UFO 3' should be considered as low acid fruits ( $\mathrm{pH}$ higher than 4.0) according to Dirlewanger et al. (2006). No consistent differences were found among rootstocks for $\mathrm{pH}$ along the study (Table 5). In contrast, SSC was affected by rootstocks. Thus, SSC was greater

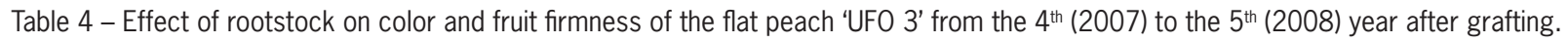

\begin{tabular}{|c|c|c|c|c|c|c|c|c|c|c|c|c|c|c|c|}
\hline \multirow{2}{*}{ Rootstock } & \multicolumn{3}{|c|}{ Fruit firmness } & \multicolumn{3}{|c|}{$a^{*}$} & \multicolumn{3}{|c|}{$b^{*}$} & \multicolumn{3}{|c|}{$a^{*} / b^{*}$} & \multicolumn{3}{|c|}{$L^{*}$} \\
\hline & 2007 & 2008 & Average & 2007 & 2008 & Average & 2007 & 2008 & Average & 2007 & 2008 & Average & 2007 & 2008 & Average \\
\hline & & & & & & & & & & & & & & & \\
\hline 677 & $4.01 \mathrm{bc}$ & $3.75 \mathrm{bcd}$ & $3.88 \mathrm{~cd}$ & $17.34 \mathrm{a}$ & $25.07 \mathrm{bc}$ & $21.21 \mathrm{ab}$ & $28.72 \mathrm{a}$ & $18.07 b$ & $23.40 \mathrm{ab}$ & $0.62 a b$ & $1.51 \mathrm{~b}$ & $1.07 \mathrm{~b}$ & $55.82 \mathrm{~d}$ & $48.22 b$ & 52.02 bc \\
\hline Krymsk $^{\circledR} 86$ & $3.86 a b c$ & $3.74 \mathrm{bcd}$ & $3.81 \mathrm{bc}$ & $24.36 d$ & $22.97 \mathrm{~b}$ & $23.66 \mathrm{~b} c$ & $31.06 \mathrm{bc}$ & $18.96 \mathrm{~b}$ & $25.01 \mathrm{ab}$ & $0.80 \mathrm{c}$ & $1.36 \mathrm{~b}$ & $1.08 \mathrm{~b}$ & $45.44 \mathrm{a}$ & $49.72 \mathrm{~b}$ & $47.58 \mathrm{a}$ \\
\hline ADAC 97-36 & $3.97 a b c$ & $3.51 a b c$ & $3.74 b c$ & $20.30 a b c$ & $25.91 \mathrm{bc}$ & $23.11 b c$ & $29.64 a b$ & $18.29 b$ & $23.96 \mathrm{ab}$ & $0.70 a b c$ & $1.57 \mathrm{bc}$ & $1.13 \mathrm{~b}$ & $51.67 \mathrm{bc}$ & $48.67 \mathrm{~b}$ & $50.17 \mathrm{ab}$ \\
\hline ADAC 99-05 & $4.17 c$ & $3.98 \mathrm{~d}$ & $4.08 \mathrm{~d}$ & $25.17 \mathrm{~d}$ & $27.39 c$ & $26.28 d$ & $32.68 \mathrm{~cd}$ & $18.37 \mathrm{~b}$ & 25.53 & $0.77 c$ & $1.61 \mathrm{bc}$ & $1.19 \mathrm{~b}$ & $49.79 \mathrm{bc}$ & $46.93 \mathrm{ab}$ & $48.36 \mathrm{a}$ \\
\hline ADAC 9 & $3.92 \mathrm{abc}$ & $3.85 \mathrm{~cd}$ & $3.89 \mathrm{~cd}$ & $22.80 \mathrm{bcd}$ & $24.86 \mathrm{bc}$ & $23.83 \mathrm{~cd}$ & $31.23 \mathrm{bc}$ & $17.43 a b$ & $24.33 \mathrm{ab}$ & $0.74 b c$ & $1.54 \mathrm{~b}$ & $1.14 b$ & $47.99 \mathrm{ab}$ & $47.64 \mathrm{ab}$ & $47.82 \mathrm{a}$ \\
\hline PADAC 0024-01 & $4.12 \mathrm{bc}$ & $3.60 \mathrm{bc}$ & $3.86 \mathrm{~cd}$ & $19.43 a b$ & $23.70 \mathrm{~b}$ & $21.56 \mathrm{abc}$ & $32.19 \mathrm{~cd}$ & $16.91 \mathrm{ab}$ & $24.55 \mathrm{ab}$ & $0.62 \mathrm{a}$ & $1.59 \mathrm{bc}$ & $1.10 \mathrm{~b}$ & $51.31 \mathrm{bc}$ & $46.32 \mathrm{ab}$ & $48.81 \mathrm{a}$ \\
\hline AC 0021-01 & $3.63 \mathrm{a}$ & $3.26 \mathrm{a}$ & $3.45 a$ & $23.04 \mathrm{~cd}$ & $15.92 \mathrm{a}$ & 19.48 a & $33.34 \mathrm{~d}$ & $24.50 \mathrm{c}$ & $28.92 \mathrm{c}$ & $0.70 a b c$ & $0.86 \mathrm{a}$ & $0.78 a$ & $49.20 \mathrm{abc}$ & $56.95 c$ & $53.08 \mathrm{c}$ \\
\hline PAC 0022-01 & $3.78 \mathrm{ab}$ & $3.44 \mathrm{ab}$ & 3.61ab & $19.90 \mathrm{abc}$ & $25.45 \mathrm{bc}$ & $22.68 \mathrm{bc}$ & $29.87 a b$ & $15.59 \mathrm{a}$ & $22.73 \mathrm{a}$ & $0.69 a b c$ & $1.80 \mathrm{c}$ & $1.24 \mathrm{~b}$ & $52.01 \mathrm{~cd}$ & $44.21 \mathrm{a}$ & $48.11 \mathrm{a}$ \\
\hline
\end{tabular}

For each character, means followed by the same letter in each column are not different (LSD test, $p<0.05$ ).

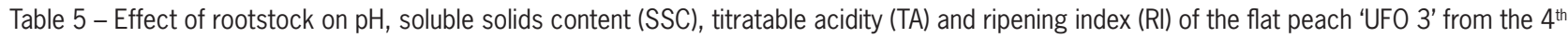
(2007) to the $5^{\text {th }}$ (2008) year after grafting.

\begin{tabular}{|c|c|c|c|c|c|c|c|c|c|c|c|c|}
\hline \multirow{2}{*}{ Rootstock } & \multicolumn{3}{|c|}{$\mathrm{pH}$} & \multicolumn{3}{|c|}{ SSC ('Brix) } & \multicolumn{3}{|c|}{ Titratable acidity (TA) } & \multicolumn{3}{|c|}{ Ripening index (RI) } \\
\hline & 2007 & 2008 & Average & 2007 & 2008 & Average & 2007 & 2008 & Average & 2007 & 2008 & Average \\
\hline GF-677 & $4.08 \mathrm{c}$ & $4.08 \mathrm{a}$ & $4.08 \mathrm{ab}$ & $14.00 \mathrm{c}$ & $12.87 \mathrm{~b}$ & $13.43 b$ & $4.55 b$ & $5.22 b$ & $4.89 \mathrm{~b}$ & $32.03 \mathrm{a}$ & $25.00 \mathrm{a}$ & $28.51 \mathrm{a}$ \\
\hline Krymsk $^{\circledast} 86$ & $4.01 \mathrm{c}$ & $4.12 \mathrm{ab}$ & $4.07 \mathrm{ab}$ & $12.30 \mathrm{ab}$ & $13.75 c$ & $13.02 \mathrm{ab}$ & $4.02 \mathrm{ab}$ & $4.58 \mathrm{ab}$ & $4.30 \mathrm{ab}$ & $30.79 a$ & $30.25 a b$ & $30.52 a b$ \\
\hline PADAC 97-36 & $3.98 \mathrm{bc}$ & $4.08 \mathrm{a}$ & $4.03 a b$ & $12.55 \mathrm{abc}$ & $14.50 \mathrm{~cd}$ & $13.52 b$ & $4.19 \mathrm{ab}$ & $5.02 a b$ & $4.61 \mathrm{ab}$ & $30.10 \mathrm{a}$ & $30.16 a b$ & $30.13 a b$ \\
\hline PADAC 99-05 & $4.10 \mathrm{c}$ & $4.11 \mathrm{ab}$ & $4.10 \mathrm{~b}$ & $12.07 \mathrm{a}$ & $14.62 \mathrm{~d}$ & $13.35 b$ & 3.68 a & $4.39 a b$ & $4.04 \mathrm{a}$ & $33.42 \mathrm{a}$ & $33.40 \mathrm{~b}$ & $33.41 \mathrm{~b}$ \\
\hline PADAC 9912-03 & $3.97 a b c$ & $4.21 \mathrm{ab}$ & $4.09 \mathrm{~b}$ & $12.60 \mathrm{abc}$ & $11.50 \mathrm{a}$ & $12.05 \mathrm{a}$ & $3.98 a b$ & $4.72 a b$ & $4.35 \mathrm{ab}$ & $31.68 \mathrm{a}$ & $24.55 \mathrm{a}$ & $28.11 \mathrm{a}$ \\
\hline PADAC 0024-01 & $3.91 \mathrm{abc}$ & 4.07 a & $3.99 a b$ & $13.45 a b c$ & $12.75 b$ & $13.10 a b$ & $4.23 \mathrm{ab}$ & $4.25 \mathrm{a}$ & $4.24 \mathrm{ab}$ & $32.12 \mathrm{a}$ & $30.08 a b$ & $31.10 a b$ \\
\hline PAC 0021-01 & $3.79 a b$ & $4.29 b$ & $4.04 \mathrm{ab}$ & $13.30 \mathrm{abc}$ & $14.12 \mathrm{~cd}$ & $13.71 b$ & $4.09 \mathrm{ab}$ & $4.78 \mathrm{ab}$ & $4.43 \mathrm{ab}$ & $32.66 \mathrm{a}$ & $29.88 a b$ & $31.27 \mathrm{ab}$ \\
\hline PAC 0022-01 & $3.77 \mathrm{a}$ & $4.05 \mathrm{a}$ & $3.91 \mathrm{a}$ & $13.65 \mathrm{bc}$ & $13.75 c$ & $13.70 \mathrm{~b}$ & $4.39 a b$ & $4.99 a b$ & $4.69 \mathrm{ab}$ & $31.08 \mathrm{a}$ & $28.12 a b$ & $29.59 \mathrm{ab}$ \\
\hline
\end{tabular}

For each character, means followed by the same letter in each column are not different (LSD test, $p<0.05$ ). SSC: soluble solids content; TA: $\mathrm{g}$ malic acid $100 \mathrm{~g}^{-1}$ fresh weight; RI: SSC/TA 
Table 6 - Pearson product moment correlations between peach physical and chemical characteristics.

\begin{tabular}{|c|c|c|c|c|c|c|c|c|c|c|c|c|c|c|c|c|}
\hline & 1 & 2 & 3 & 4 & 5 & 6 & 7 & 8 & 9 & 10 & 11 & 12 & 13 & 14 & 15 & 16 \\
\hline 1. Fruit weight & 1 & & & & & & & & & & & & & & & \\
\hline 2. Equatorial diameter & 0.7588 & 1 & & & & & & & & & & & & & & \\
\hline 3. Flesh firmness & ns & ns & 1 & & & & & & & & & & & & & \\
\hline 4. Pulp thickness & ns & ns & ns & 1 & & & & & & & & & & & & \\
\hline 5. $L^{*}$ & ns & ns & ns & ns & 1 & & & & & & & & & & & \\
\hline 6. $a^{*}$ & ns & ns & 0.5981 & ns & -0.8385 & 1 & & & & & & & & & & \\
\hline 7. $b^{*}$ & ns & ns & ns & ns & 0.9800 & -0.7757 & 1 & & & & & & & & & \\
\hline 8. $a^{*} / b^{*}$ & ns & ns & ns & ns & -0.9646 & 0.9306 & -0.9318 & 1 & & & & & & & & \\
\hline 9. $\mathrm{pH}$ & ns & ns & ns & ns & ns & ns & ns & ns & 1 & & & & & & & \\
\hline 10. TA & ns & ns & ns & ns & ns & ns & ns & ns & ns & 1 & & & & & & \\
\hline 11. SSC & ns & -0.5194 & ns & ns & ns & ns & ns & ns & ns & ns & 1 & & & & & \\
\hline 12. RI & ns & -0.5420 & ns & ns & ns & ns & ns & ns & ns & -0.8432 & 0.6418 & 1 & & & & \\
\hline 13. TCSA & 0.4885 & ns & ns & ns & ns & ns & ns & ns & ns & ns & ns & ns & 1 & & & \\
\hline 14. Yield & ns & ns & ns & ns & ns & ns & ns & ns & ns & ns & ns & ns & ns & 1 & & \\
\hline 15. Cumulative yield & 0.5421 & 0.5964 & ns & ns & ns & ns & ns & ns & ns & ns & ns & ns & ns & ns & 1 & \\
\hline 16. Yield efficiency & ns & ns & ns & ns & ns & ns & ns & ns & ns & ns & ns & ns & 0.5913 & ns & ns & 1 \\
\hline
\end{tabular}

Significant ( $p<0.05)$; ns: not significant.

on PAC 0021-01, PAC 0022-01, GF-677, PADAC 97-36 and PADAC 99-05 and the differences were significant when compared with PADAC 9912-03. With the exception of PADAC 9912-03 and Krymsk ${ }^{\circledR}$ 86, SSC values were similar to those reported by Nicotra et al. (2001) for this cultivar. It is well known the influence of rootstock on SSC, as it has been previously reported for peaches (Albás et al., 2004; Tsipouridis and Thomidis, 2005), cherries (Cantín et al., 2010; Moreno et al., 2001) and plums (Daza et al., 2008).

Regarding titratable acidity (TA), small but not consistent differences were found among rootstocks on the two years of study. In average, the lowest TA was recorded in 'UFO 3' trees grafted on PADAC 99-05, and the higher on GF-677, while no differences were found among all the other rootstocks. In average, fruits of 'UFO 3' (Table 5) showed a tendency to have higher RI when grafted on PADAC 99-05.

The interdependence of the variables was investigated by the analysis of correlation (Table 6). Sixteen pairs of variables were highly correlated. As expected, correlation analyses showed the higher correlation values between fruit weight and equatorial diameter $(\mathrm{r}=$ $0.76, p \leq 0.05$ ), as well as between fruit weight and TCSA $(r=0.49)$. This would indicate that tree vigor is affecting fruit size. The skin brightness was negatively correlated with skin redness ( $\mathrm{a}^{*}$ values) $(\mathrm{r}=-0.84)$ and $\mathrm{a}^{*} / \mathrm{b}^{*}$ ratio $(\mathrm{r}=-0.96)$, while it was positively correlated with skin yellowness $(r=0.98)$. Similar results were reported for other clingstone peaches (Drogoudi and Tsipouridis, 2007). Skin redness was positively correlated with relation $\mathrm{a}^{*} / \mathrm{b}^{*}(\mathrm{r}=0.93)$, while the skin yellowness was negatively correlated with $\mathrm{a} * / \mathrm{b} *$ ratio $(\mathrm{r}=$ -0.93). Skin redness was positively correlated with flesh firmness $(\mathrm{r}=0.59)$, these two characteristics widely appreciated in peach flat cultivars by consumers. These results are similar to those obtained by Orazem et al. (2011).

\section{Conclusions}

The peach-almond hybrid GF-677 resulted in the most invigorating rootstocks for 'UFO 3' production in our growing conditions. The size-controlling properties of PADAC 99-05 could be considered of high interest for reducing production cost, particularly pruning and harvest, due to smaller tree size.

\section{Acknowledgements}

To Selecplant, S.L. (Murcia, Spain) for providing an experimental site and maintain the trial in optimum conditions.

\section{References}

Albás, E.S.; Jiménez, S.; Aparicio, J.; Betrán, J.A.; Moreno, M.A. 2004. Effect of several peach $\times$ almond hybrids rootstocks on fruit quality of peaches. Acta Horticulturae 658: 321-326.

Bielicki, P.; Czynczyk, A.; Chlebowska, D. 2000. Effect of a rootstock and tree location on yield and fruit quality of 'King Jonagold' apples. Journal of Fruit and Ornamental Plant Research 8: 65-71.

Bielicki, P.; Czynczyk, A.; Chlebowska, D. 2004. Effect of several new polish rootstocks and M9 subclones on growth, yield and fruit quality of two apple 'King Jonagold' and 'Elshof' cultivars. Acta Horticulturae 658: 327-332.

Byrne, D.H. 2002. Peach breeding trends. Acta Horticulturae 592: 49-59.

Cantín, C.M.; Gogorcena, Y.; Moreno M.A. 2009. Analysis of phenotypic variation of sugar profile in different peach and nectarine [Prunus persica (L.) Batsch] breeding progenies. Journal of the Science of Food and Agriculture 89: 1909-1917.

Cantín, C.M.; Pinochet, J.; Gogorcena, Y.; Moreno, M.A. 2010. Growth, yield and fruit quality of 'Van' and 'Stark Hardy Giant' sweet cherry cultivars as influenced by grafting on different rootstocks. Scientia Horticulturae 123: 329-335. 
Caruso, T.; Giovanni, D.; Liverani, A. 1996. Rootstock influences the fruit mineral, sugar and organic acid content of a very early ripening peach cultivar. Journal of Horticultural Science 71: 931-937.

Caruso, T.; Inglese, P.; Sidari, M.; Sottile, F. 1997. Rootstock influences seasonal dry matter and carbohydrate content and partitioning in above-ground components of 'Flordaprince' peach trees. Journal of the American Society for Horticultural Science 122: 673-679.

Cevallos-Casals, B.A.; Byrne, D.H.; Okie, W.R.; Cisneros-Zevallos, L. 2006. Selecting new peach and plum genotypes rich in phenolic compounds and enhaced functional properties. Food Chemistry 96: 273-280.

Chun, J.; Fallahi, E.; 2001. The influence of spacing and rootstock on foliar mineral nutrition and fruit quality of 'Fuji' apple trees. Journal of the Korean Society for Horticultural Science 42: 621-624.

Cinelli, F.; Loreti, F. 2004. Evaluation of some plum rootstocks in relation to lime-induced chlorosis by hydroponic culture. Acta Horticulturae 658: 421-428.

Crisosto, C.H.; Crisosto, G.M.; Metheney, P. 2003. Consumer acceptance of 'Brooks' and 'Bing' cherries is mainly dependent on fruit SSC and visual skin color. Postharvest Biology \& Technology 28: 159-167.

Daza, A.; García-Galavís, P.A.; Grande, M.J.; Santamaría, C. 2008. Fruit quality parameters of 'Pioneer' Japanese plums produced on eight different rootstocks. Scientia Horticulturae 118: 206211.

Dichio, B.; Xiloyannis, C.; Celano, G.; Vicinanza, L.; Gómez-Aparisi, J.; Esmenjaud, D.; Salesses, G. 2004. Performance of new selections of Prunus rootstocks resistant to Root Knot nematodes, in water logging conditions. Acta Horticulturae 658: 403406.

Dirlewanger, E.; Cosson, P.; Boudehri, K.; Renaud, C.; Capdeville, G.; Tauzin, Y.; Laigret, F.; Moing, A. 2006. Development of a second-generation genetic linkage map for peach [Prunus persica (L.) Bastch] and characterization of morphological traits affecting flower and fruit. Tree Genetics \& Genomes 3: 1-13.

Drogoudi, P.D.; Tsipouridis, C.Gr. 2007. Effects of cultivar and rootstock on the antioxidant content and physical characters of clingstone peaches. Scientia Horticulturae 115: 34-39.

Food and Agriculture Organization of the United Nations. [FAO]. STATistics 2009. Available at: http://faostat.fao.org/site/339/default.aspx [Accessed Jan. 10, 2011].

Giorgi, M.; Capocasa, F.; Scalzo, J.; Murri, G.; Battino, M.; Mezzetti, B. 2005. The rootstock effects on plant adaptability, production, fruit quality, and nutrition in the peach (cv. 'Suncrest'). Scientia Horticulturae 107: 36-42.

Gonçalves, B.; Moutinho-Pereira, J.; Santos, A.; Silva, A.P.; Bacelar, E.; Correia, C.; Rosa, E. 2006. Scion-rootstock interaction affects the physiology and fruit quality of sweet cherry. Tree Physiology 26: 93-104.

Hudina, M.; Fajt, N.; Stampar, F. 2006. Influence of rootstock on orchard productivity and fruit quality in peach cv. 'Redhaven'. Journal of Horticultural Science \& Biotechnology 81: 10641068 .
Iglesias, I.; Monserrat, R.; Carbó, J.; Bonany J.; Casals, M. 2004. Evaluation of agronomical performance of several peach rootstocks in Lleida and Girona (Catalonia, NE-Spain). Acta Horticulturae 658: 341-348.

Iglesias, I.; Echeverría, G. 2009. Differential effect of cultivar and harvest date on nectarine colour, quality and consumer acceptance. Scientia Horticulturae 120: 41-50.

Ministerio de Medio Ambiente y Medio Rural y Marino [MARMM]. 2009. Statistical yearbook. 2009. Available at: http/ www.mapa.es/es/estadistica/pags/anuario/2009/indice.asp?part $\mathrm{e}=3 \&$ capitulo $=13 \&$ grupo $=9 \&$ seccion $=10$ [Accessed Dec. 22, 2010] (in Spanish).

Moreno, M.A.; Adrada, R.; Aparicio J.; Betrán J. A. 2001. Performance of 'Sunburst' sweet cherry grafted on different rootstocks. Journal of Horticultural Science \& Biotechnology 76: 167-173.

Nicotra, A.; Conte, L. 2003. New fruit typology for the peach market: birth of the 'Ufo' and 'Ghiaccio' series. Revista di Fruticoltura e di Ortofloricoltura 65: 20-25.

Nicotra, A.; Conte, L.; Moser, L.; Fantechi, P. 2001. The Ufo series, six new cultivars of peach with flat fruit. Informatore Agrario 50: 61-64.

Orazem, P.; Stampar, F.; Hudina, M. 2011. Quality analysis of 'Redhaven' peach fruit grafted on 11 rootstocks of different genetic origin in a replant soil. Food Chemistry 124: 1691-1698.

Remorini, D.; Tavarini, S.; Degl'Innocenti, E.; Loreti, F.; Massai, R.; Guidi, L. 2008. Effect of rootstocks and harvesting time on the nutritional quality of peel and flesh of peach fruits. Food Chemistry 110: 361-367.

Taylor, M.A.; Rabe, E.; Dodd, M.C.; Jacobs, G. 1993. Influence of sampling date and position in the tree on mineral nutrients, maturity and gel breakdown in cold stored 'Songold' plums. Scientia Horticulturae 54: 131-141.

Tsipouridis, C.Gr.; Thomidis, T. 2005. Effect of 14 peach rootstocks on the yield, fruit quality, mortality, girth expansion and resistance to frost damages of 'May Crest' peach variety and their susceptibility to Phytophthora citrophthora. Scientia Horticulturae 103: 421-428.

Union for the Protection of New Varieties of Plants [UPOV]. 1995. Guidelines for the conduct of tests for distinctness, uniformity and stability. Available at: http://www.upov.int/en/publications/ tg_rom/ [Accessed Aug. 15, 2010].

Zarrouk, O.; Gogorcena, Y.; Gómez-Aparisi, J.; Betrán, J.A.; Moreno, M.A. 2005. Influence of almond $\times$ peach hybrids rootstocks on flower and leaf mineral concentration, yield and vigour of two cultivars. Scientia Horticulturae 106: 502-514.

Zarrouk, O.; Gogorcena, Y.; Moreno, M.A.; Pinochet, J. 2006. Graft compatibility between peach cultivars and Prunus rootstocks. HortScience 41: 1389-1394. 